Ann. Biol. anim. Bioch. Biophys, 1975, 15 (3), 607-609.

NOTE

\title{
RÔLE DE LA DURÉE QUOTIDIENNE D'ÉCLAIREMENT SUR LA RÉPONSE, APRES HÉMIGASTRATION, DU TESTICULE RESTANT CHEZ LE CAMPAGNOL DES CHAMPS (MICROTUS ARVALIS)
}

\author{
Lise MARTINET et Monique MEUNIER
}

Station centrale de Physiologie animale,

Centre national de Recherches zootechniques, I. N.R. A., ;8350 Jouy en Josas

Il est généralement admis sur la base de l'observation et de l'expérimentation que le rythme photopériodique est un des facteurs déterminant du cycle annuel de reproduction du Campagnol (LecyK, I962 ; Bernard, I964; Martinet, I966).

D'autres recherches ont conduit à penser que la qualité de la végétation consommée et la température jouaient également un rôle important dans la régulation de ce cycle (MARTINet et Meunier, i969; Daketse, 1974).

Mais les conclusions de Delost (I970, 1972) ne confirment pas le rôle que nous attribuons à ces deux facteurs. Nous avons donc repris la technique utilisée par cet auteur, à savoir l'hémicastration, pour comprendre si elle pouvait modifier le rôle des facteurs externes sur la reproduction.

\section{MATÉRIEI, ET MÉTHODES}

Des campagnols mâles sont placés dès leur naissance sous une photopériode claire quotidienne de 15 heures ou ro heures.

A 45 jours, une hémicastration est pratiquée sur la moitié des mâles de chacun des deux lots. Les animaux sont tués à go jours, les testicules pesés, les réserves épididymaires évaluées selon la technique précédemment décrite (Martinet, 1966).

\section{RÉSUITAT ET DISCUSSION}

Si l'on compare, pour une même durée d'éclairement, les animaux témoins et les animaux hémicastrés on observe une hypertrophie du testicule restant chez les hémicastrés (tabl. I).

Cette hypertrophie est beaucoup plus importante sous io heures que sous I $_{5}$ heures d'éclai- 
rement quotidien de telle sorte que l'influence de la durée de la photopériode claire hautement significative chez les animaux témoins, est en partie masquée chez les animaux hémicastrés.

Les résultats obtenus chez le Bélier sont tout à fait comparables (Hochereau de Reviers et Pelletier, I971).

\section{TABLEAU I}

Influence de l'hémicastration sur les testicules et les réserves épididymaires

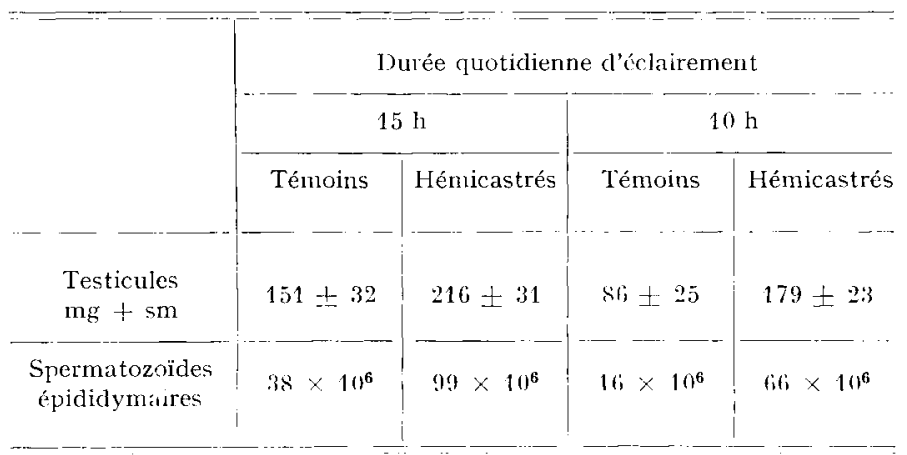

La méthode de l'hémicastration, utilisée pour comparer une croissance de testicules avant et après traitement est donc très discutable pour plusieurs raisons :

- L'hypertrophie compensatrice qui se produit est variable avec l'âge de l'animal, l'intervalle de temps séparant l'hémicastration de l'autopsie (Hochereau de Reviers, r97r) et ici le traitement appliqué comme le montre la différence obtenue avec deux photopériocles claires.

-- L'hémicastration modifie le niveau de stéroïdes circulants; or on sait que les facteurs externes agissent en grande partie en modifiant la sensibilité du système nerveux central à la rétroaction négative des stéroïdes (Pelletier et Ortavant, 1975).

Reçu pour publication en février 1975.

\section{SUMMARY}

\section{ROI, OF DAYLIGH'T RATIO IN RESPONSE OF THE REMAINING TESTIS OF THE HEMICASTRATED FIELL-VOLE (MICROTUS ARVALIS)}

Compensatory hypertrophy of the testis remaining after hemicastration is controlled by the length of the daylight period.

Thus, this method cannot be used to compare testis weights before and after hemicastration in field-vole in various light rythms.

\section{RÉFÉRENCES BIBLIOGRAPHIQUES}

Bernard J., x964. Note préliminaire sur la zeproduction chez le Campagnol des champs dans le sud de la Belgique. Bull. Inst. A gron. Sta. Reci.. Gemblous, 32, 275-289.

Daketse M. J., I974. Influence de la température sur la mortalité juvénile, la croissance et la fertilité du Campagnol des champs. Thèse $3^{\mathrm{e}}$ cycle Fac. Sci., Paris VI, $4 \mathrm{I}$ p. 
Delost P., r97o fitude expérimentale des causes du repos hivernal chez jes manmifères à cycle sexuel saisonnier. C. R. Soc. Biol., 164, $2475-2479$.

Delost P., I972. Étude expérimentale de la reprise printanière de l'activité sexuelle chez les mammifères à cycle reproducteur saisonnier. C.R. Soc. Biol., 166, 879-884.

Hochereau de Reviers M. T., Ig7r. Action de l'hémicastration sur l'évolution du testicule restant chez le Rat. Bull. Assoc. Anat., 151, 364-37I.

Hochereau de Reviers M. T., Pelletifr J., ig7i. Unilateral castration in the ram. J. Reprod. Fert., 2\%, 498 (abst.).

Lecyck M., r962. The effect of the length of daylight on reproduction in the field vole (Microtus arvalis). Zool. Pol, 12, 189-22I.

Martinet L., I966. Modification de la spermatogenèse chez le Campagnol des champs (Microtus arvalis) en fonction de la durée quotidienne d'éclairement. Ann. Biol. anim. Bioch. Biophys., 6, 30I-3I3.

Martinet L., Meunier M., 1969. Influence des variations saisonnières de la Luzerne sur la croissance, la mortalité et la reproduction du Campagnol des champs. Ann. Biol. anim. Bioch. Biophys., 9, 45I-46z.

Pelletier J., Ortavant R., 1975. Photoperiodic control of LH release in the ram. II. Lightandrogens interaction. Acta endocrin., 3, 442-450. 